\title{
Case Report: A Rare Case of Penetrating Trauma of Frontal Sinus with Anterior Table Fracture
}

\author{
Himanshu Raval ${ }^{*}$, Mona Bhatt ${ }^{2}$ and Nihar Gaur ${ }^{3}$ \\ ${ }^{1}$ Department of Neurosurgery, NHL Municipal Medical College, SVP Hospital Campus, Gujarat, India \\ ${ }^{2}$ Medical Officer, CHC Dolasa, Gujarat, India \\ ${ }^{3}$ GAIMS-GK General Hospital, Gujarat, India
}

*Corresponding author: Dr. Himanshu Raval, Resident, Department of Neurosurgery, NHL Municipal Medical College, SVP Hospital Campus, Elisbridge, Ahmedabad, Gujarat, 380006, India, Tel: 942-955-3329

\begin{abstract}
Background: Head injury is common component of any road traffic accident injury. Injury involving only frontal sinus is uncommon and unique as its management algorithm is changing over time with development of radiological modalities as well as endoscopic intervention. Frontal sinus injuries may range from isolated anterior table fractures causing a simple aesthetic deformity to complex fractures involving the frontal recess, orbits, skull base, and intracranial contents. Only anterior table injury of frontal sinus is rare in penetrating head injury without underlying brain injury with history of unconsciousness and questionable convulsion which is new to existing literature.
\end{abstract}

Case description: A 72-years-old patient came with the history of road traffic accident and a piece of wooden block penetrating forehead. Though there is no apparent neurological deficit on time of presentation but brief episode of unconsciousness and questionable convulsion activity just after injury raise the question of possible brain insult and requirement of cranial opening. With availability of CT scan, extent of injury was measured. Cranialization, obliteration, reconstruction, observation, and endoscopic sinus surgery are available treatment modalities. Patient was treated with open reduction and internal fixation. There is no post-operative complication in subsequent follow-up.

Conclusion: The first impression that come across mind of surgeon at trauma bay that penetrating head injury in frontal region with history of unconsciousness most likely to cause brain injury, this case shows no cranialization/craniotomy is required in every case of penetrating injury and careful decision making save patient from post-operative morbidity.

\section{Keywords}

Head injury, Frontal sinus, Outer table, Fracture

\section{Introduction}

Road traffic accident (RTA) is the most common cause of cranio-facial injury and involvement of frontal bone fractures are rare and constitute $5-9 \%$ of only facial trauma. The degree of association has been reported to be $95 \%$ with fractures of the anterior table or wall of the frontal sinuses, $60 \%$ with the orbital rims, and $60 \%$ with complex injuries of the naso-orbital-ethmoid region, 33\% with other orbital wall fractures and $27 \%$ with Le Fort level fractures. Blunt trauma is commonest to involve frontal sinus [1]. Very few literatures available to enlighten the penetrating trauma and its extent wise management. Consultation with neurosurgeon is required with priority in case of injury involving any part of skull bone. Radiological imaging plays important role in accurate diagnosis and decision making of further management. Different specialty branch have their way to manage frontal sinus injury. Neurosurgical complications may present acutely or may have a delayed presentation. In the acute period, the main concern is cerebrospinal fluid (CSF) leakage, with risk of subsequent seeding of infection and progression to meningitis or encephalitis. Delayed complications include brain abscess and mucocele formation. Mucocele formation, which may result from obstruction of the frontal sinus egress or direct trauma to the frontal sinus mucosa, may progress to a mucopyocele [2]. Significant intracranial injury occurs more commonly with injury to the frontal sinus (12-17\% of the time) than with injury to the

Citation: Raval H, Bhatt M, Gaur N (2020) Case Report: A Rare Case of Penetrating Trauma of Frontal Sinus with Anterior Table Fracture. Neurosurg Cases Rev 3:046. doi.org/10.23937/26434474/1710046

Accepted: November 23, 2020; Published: November 25, 2020

Copyright: (c) 2020 Raval $\mathrm{H}$, et al. This is an open-access article distributed under the terms of the Creative Commons Attribution License, which permits unrestricted use, distribution, and reproduction in any medium, provided the original author and source are credited. 
mandible or mid face due to the proximity of the frontal sinus to the brain and the great forces required to cause a frontal sinus fracture [3].

The uniqueness of this case report is patient's presentation at trauma bay which include following: presence of intact foreign body in penetrating wound (which is not reported yet), no neurological deficit with history of unconsciousness and questionable convulsion.

\section{Case Report}

Following case report is noted as per CARE guideline. Proper consent has been taken from patient in her native language. A 72-years-old female admitted in SVP hospital in April, 2020 with complaint of forehead injury and brief episode of unconsciousness due to collision of bike with a tree, while she was a pillion rider. There were no signs and symptoms of raised intracranial pressure such as vomiting, difficulty in breathing and altered consciousness but there was history of unconsciousness and questionable convulsion which warrants neurosurgical attention. There was no leakage from nasal cavity as well as from both ears. She was having no co-morbidities and no previous operative history. She was conscious, oriented and co-operative at time of presentation. On neurological examination, her Glasgow coma scale was $15 / 15$, cranial nerve examination was normal and no abnormalities were found in cerebellar examination. Power and tone were normal. There was no paresis, reflexes were normal. Sensory examination was normal. She was having no icterus, pallor, clubbing, cyanosis, edema and lymphadenopathy. On local examination there was a piece of wood which was piercing through her forehead just above medial end of left supraorbital ridge. Dimension of entry wound was approximately $3 \times 3 \mathrm{~cm}$ size with oozing of blood at margins. There was slight edema surrounding it. There was no raccoon eye sign bilaterally. All routine investigations were done and were within normal limits. Ct brain was done. It was showing fracture of anterior table of left frontal sinus with presence of foreign body that had pierced through the wound. All pre-operative workup were done and the patient had giver consent after being informed of the risks of bleeding, infection, paresthesia, brain injury, CSF leak, meningitis, diplopia, visual loss, external deformity, and late mucocele formation. Then patient is shifted to operative room. Coronal flap is used and the full pericranial exposure was maintained. After full exposure foreign body carefully removed and thorough examination at local site was done. Fragments of anterior table were carefully removed and kept moist. Mucosa of frontal sinus is denuded from posterior table and cavity is examined for any CSF leak with concurrent Valsalva maneuver. Then cavity is cauterized and a small frontalis muscle plug is then placed into the frontal recess to obliterate the ostia. A fat graft is obtained through a left lower-quadrant (or periumbilical) incision using a separate, sterile instrument set. Fat

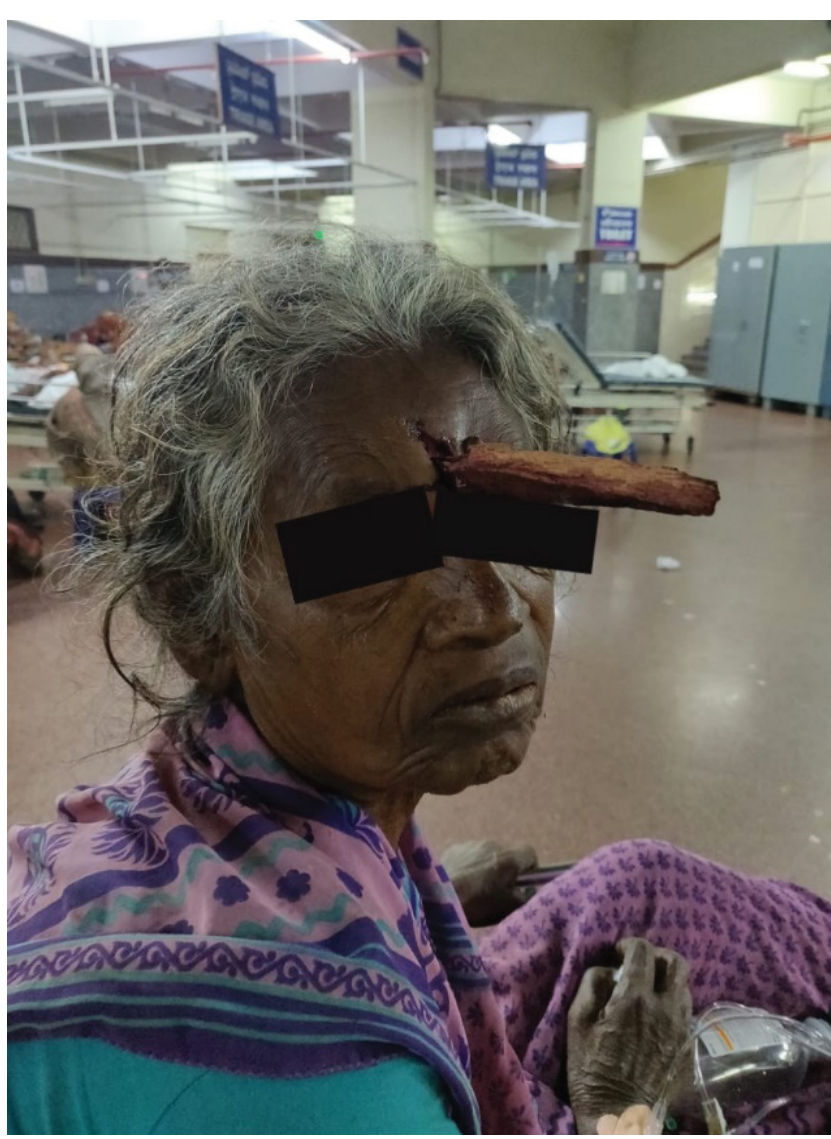

Figure 1: Penetrating injury over frontal region.

graft was obtained in single attempt with minimal surrounding trauma. The fat graft was then trimmed and inserted into the sinus cavity to exclude the possibility of future development of CSF fistula. The anterior table fragments are replaced. Anterior table stabilization is achieved with micro plates, and bone plate described as "Open Reduction and Internal Fixation". Skin and soft tissue at local wound were approximated with non-absorbable suture material and sterile dressing applied. Coronal flap reposition carefully without development of tension in suture line. There was no post-operative leakage from wound or there was no nasal watery discharge. During post-operative period, patient's neurological examination was normal and there was no nasal discharge. She was treated with antibiotics, analgesics and anti-epileptics (it was for questionable convulsion that patient had after injury) during post-operative period. Patient was discharged on $3^{\text {rd }}$ post-operative day. During follow up, suture were removed. There was no any sign and symptoms suggestive of CSF rhinorrhea and convulsion over 3 months of follow-up (Figure 1 and Figure 2).

\section{Discussion}

Term craniofacial territory comprise of region between coronal suture and chin. The causes of craniofacial trauma reflect the general pattern of neurotrauma but with significant regional variations. Globally, road traffic accidents are the prime reason of neurotrauma and craniofacial trauma. In developed countries, an 


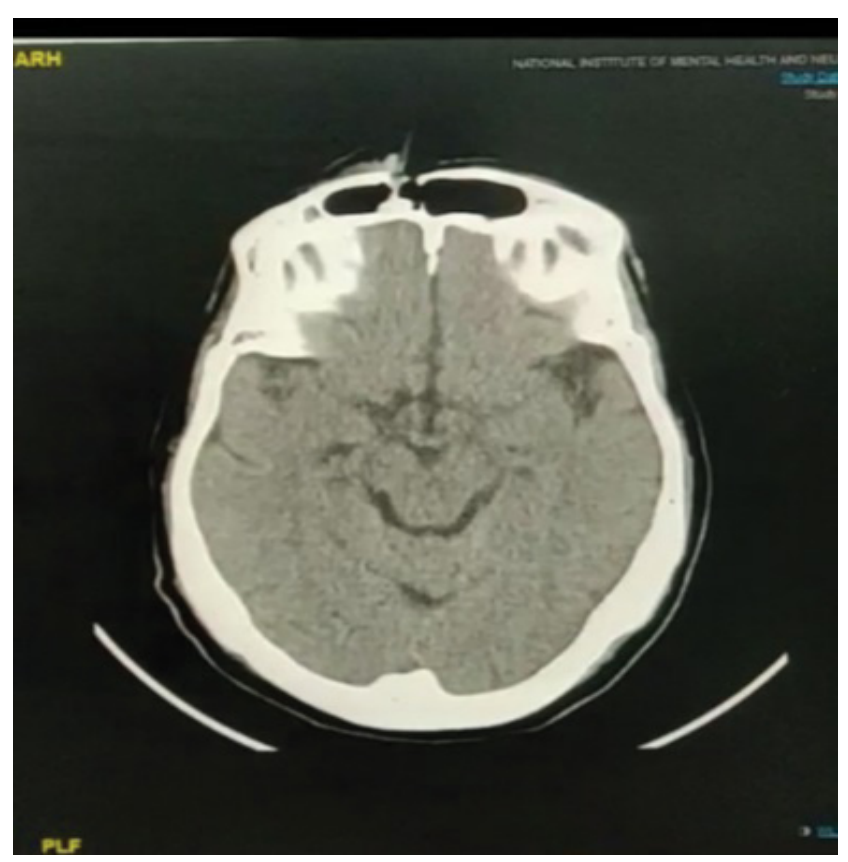

Figure 2: Axial cut showing anterior table fracture.

overall drop in transport injuries, the use of air bags in motor vehicles, and the use of helmets by motorcyclists have reduced the incidence of maxillofacial injury. Falls in infancy and old age have become major causes in many countries [4]. Though Primary mode of injury is blunt trauma, this case is also caused by RTA but the difference is patient is presented with intact foreign body pierced through her head. Usually the area over frontal sinuses is very well protected as due to presence of relatively thicker cortical bone in comparison of other regions [5]. Frontal sinus injury is common in patients who have experienced trauma. Various treatment algorithms have been proposed, but there is little neurosurgical literature to guide treatment strategies. Severe infectious and structural complications occur if there is mismanagement of frontal sinus fractures [2]. In this case absence of any neurological sign and symptoms were striking feature as well as relieving factor but history of unconsciousness and convulsion creates confusion and attract attention toward organic insult. Usually penetrating trauma of skull bones are associated with brain injury. To rule out brain damage, a radiological investigation plays an important role. A thin-cut (1.0 to $1.5 \mathrm{~mm}$ ) axial, coronal, and sagittal computed tomography (CT) scan is the radiological gold standard for diagnosis of frontal sinus fractures. Information about the anterior and posterior tables are obtained via axial cuts. Coronal images can assess the sinus floor and orbital roof. Sagittal reconstructions can be useful in assessing the patency of the frontal recess, and three-dimensional reconstructions are outstanding to provide exact visualization of bony injury [6].

Surgical treatment depends on wide exposure of the craniofacial skeleton via a bicoronal scalp flap or periorbital, intraoral, and extraoral incisions. Here involvement of only frontal sinus warrants bicoronal flap ex- posure. Line of treatment can be decided by assessing an anterior table fracture, a posterior table fracture, a nasofrontal recess fracture, a dural tear (CSF leak), and fracture displacement/comminution and as well as degree of displacement $[7,8]$. In this case there is only breach of anterior table that we can see in axial cut. There is little risk of morbidity with non-displaced anterior table fractures. Fractures having displacement of 2 to $6 \mathrm{~mm}$ present has risk of mucocele formation. Post-operative deformity chances increases with the degree of fracture displacement. An endoscopic repair may be possible if there is no wound over skin covering sinus site and posterior wall involved. Endoscopic treatment requires experience of surgeon and his expertise with endoscope [9]. This avoids the need for a coronal incision and also allows the patient to assess the degree of deformity after all facial edema has resolved. Patient gets two scars in this case, one over local trauma site and other of incision site. More complex anterior table fractures and those that extend below the orbital rim may require open reduction using a coronal incision. Obliteration of the frontal sinus with fat grafts through a frontal osteoplastic flap in patients with chronic sinusitis and mucocele formation has been applied by some to the treatment of frontal sinus fractures [10]. In this case we have done open reduction with internal fixation. Though there was unconsciousness and possibility of convulsion during post-traumatic phase, sudden hypotension following trauma or coupe injury might be the cause. Hence, we kept patient on levetiracetam during post-operative phase [11].

\section{Conclusion}

This is a case report of an uncommon frontal bone fracture involving only anterior wall of frontal sinus due to penetrating injury with unconsciousness and questionable convulsion. The management of frontal sinus fractures by neurosurgeons can be different based on extent of injury. One should not only rely on external appearance of wound as well as clinical picture, it may mislead the diagnosis and further management. History of unconsciousness is not thumb rule for brain insult and cranial opening is not the answer whenever you see penetrating head injury. However, the importance of three dimensional imaging modality can be exceptional which has led to accurate diagnosis and helpful in deciding management. The aim of treatment modality is to restoration of normal anatomy with preservation of all possible cosmetic aspect of face and prevention of all possible complication like meningitis, CSF rhinorrhea, osteomyelitis as well as local infection. With possibility of various treatment modalities, one must decide on the basis of extent of injury, its outcome and patient's economic condition and no premature decision of emergency craniotomy/craniectomy is considered.

\section{Author Contribution}

Dr. Himanshu Raval: Data acquisition and analysis, 
drafting, revision of manuscript.

Dr. Mona Bhatt: Drafting, revision of the manuscript.

Dr. Nihar Gaur: Drafting, revision of the manuscript.

\section{Conflict of Interest}

None.

\section{Acknowledgement}

None.

\section{Funding}

None.

\section{References}

1. Taylor P McGuire, Petrus P Gomes, Cameron ML Clokie, George KB Sandor (2006) Fractures of the supraorbital rim: Principles and management. J Can Dent Assoc 72: $537-$ 540.

2. Ravindra VM, Neil JA, Shah LM, Schmidt RH, Bisson EF (2015) Surgical management of traumatic frontal sinus fractures: Case series from a single institution and literature review. Surg Neurol Int 6: 141.

3. AJ Duvall, DP Porto, D Lyons, LR Boies (1987) Frontal si- nus fractures: Analysis of treatment results. Arch Otolaryngol Head Neck Surg 113: 933-935.

4. lida S, Hassfeld S, Reuther T, Hans-Gert, Claudia H, et al. (2003) Maxillofacial fractures resulting from falls. J Craniomaxillofac Surg 31: 278.

5. Nahum AM (1975) The biomechanics of maxillofacial trauma. Clin Plast Surg 2: 59-64.

6. Hemmy DC, David DJ, Herman GT (1983) Three-dimensional reconstruction of craniofacial deformity using computed tomography. Neurosurgery 13: 534.

7. Gruss JS (1990) Complex craniomaxillofacial trauma: Evolving concepts in management. A trauma unit's experience-1989 Fraser B Gurd lecture. J Trauma 30: 377.

8. David DJ (1974) Exploration of the orbital floor through a conjunctival approach. Aust N Z J Surg 44: 25.

9. Strong EB, Buchalter GM, Moulthrop TH (2003) Endoscopic repair of isolated anterior table frontal sinus fractures. Arch Facial Plast Surg 5: 514-521.

10. Bosley WR (1972) Osteoplastic obliteration of the frontal sinus laryngoscope. Laryngoscope 82: 1463-1476.

11. Jones KE, Puccio AM, Harshman KJ, Bonnie F, Neal B, et al. (2008) Levetiracetam versus phenytoin for seizure prophylaxis in severe traumatic brain injury. Neurosurg Focus. 